Pacific Journal of Mathematics

-GENERATOR IDEALS IN PRÜFER DOMAINS 


\title{
n-GENERATOR IDEALS IN PRÜFER DOMAINS
}

\author{
RICHARD G. SWAN
}

\begin{abstract}
Heitmann has shown that a finitely generated ideal in a Prüfer domain of Krull dimension $n$ needs at most $n+1$ generators. I will show here that this result is, in fact, the best possible. The same is true for Heitmann's stability theorems.
\end{abstract}

These results, in particular, give additional counterexamples to the old question of whether a finitely generated ideal in a Prüfer domain can be generated by 2 elements [GH]. ${ }^{1}$ The first such example was given by Schülting [S] who found a 2-dimensional Prüfer domain with an ideal requiring 3 generators. His example is included in those discussed here, although the method of proof is very different.

A Prüfer domain may be characterized as a commutative integral domain in which every finitely generated ideal is invertible. Equivalently, it is a commutative integral domain $R$ such that the localization $R_{P}$ at any prime ideal is a valuation ring [KC, Th. 64]. A very thorough discussion of Prüfer rings is given in [G]. A noetherian Prüfer domain is a Dedekind ring, so it is natural to ask whether a Prüfer ring has properties similar to those of a Dedekind ring. The 2 generator question presumably first arose in this way. In some respects, a Prüfer ring behaves very much like a Dedekind ring. For example, every finitely generated torsion-free module is projective and a direct sum of ideals [CE, Ch. I, Prop. 6.1]. In addition, cancellation holds for such modules [KA, p. 75]. The results obtained here, however, show that in other aspects, a Prüfer ring behaves like a general commutative domain.

Let $\mu(M)$ denote the least number of generators of a module $M$.

THEOREM 1. For any integer $n \geq 1$, there is a Prüfer domain $R$ of Krull dimension $n$ and an ideal $I_{n}$ of $R$ with $\mu\left(I_{n}\right)=n+1$.

THEOREM 2. There is a Prüfer domain $R$ such that for every integer $n \geq 0$ there is an ideal $I_{n}$ of $R$ with $\mu\left(I_{n}\right)=n+1$.

\footnotetext{
${ }^{1}$ The question was first raised by Gilmer around 1964.
} 
The construction of these examples is quite elementary but the proof that $\mu\left(I_{n}\right)=n+1$ requires topological methods. Aside from the theory of Stiefel-Whitney classes used in [SV], the main topological fact needed is the existence of a fundamental class for algebraic spaces proved by Borel and Haefliger $[\mathbf{B H}]$. The results of $[\mathbf{B H}]$ are considerably more general and the proofs assume familiarity with sheaves and the Borel-Moore homology theory. In order to make the present paper more accessible to non-topologists, I have avoided using the results of $[\mathbf{B H}]$ and have instead repeated the essential steps of the proof in Lemmas 5, 6, 8, 10, and 11, following the suggestions in [BH, §3.8]. Thus only a knowledge of the basic properties of Čech cohomology [ES] will be needed. An algebraic approach to the homology theory of real algebraic varieties has been developed by Delfs and Knebusch [DK]. Gilmer [GG] has given a very simple proof of the result of [SV, Example 2]. However, I see no obvious way to extend this argument to cover the cases discussed here.

In $\S 5$ I will show that Heitmann's stability results for Prüfer rings are also the best possible. Finally, in $\S 6$, I will discuss the relation to Schülting's example.

I would like to thank R. Gilmer, R. Heitmann, I. Kaplansky, and T.-Y. Lam for suggesting a number of improvements to an earlier version of this paper and for references to previous work on the problem.

1. The algebraic construction. The basic construction was introduced by Dress [D] and generalized by Gilmer [G2]. A modified form of it was used by Schülting [S]. Further discussion is given in $\S 6$. The construction is based on the following lemma, a variant of a result of Dress [D]. It gives a sufficient condition for a ring to be a Prüfer ring. The condition is not necessary, e.g., let $R$ be $\mathbf{Z}$ or a polynomial ring over a field. The last statement in the lemma was pointed out to me by Heitmann.

LEMMA 1. Let $R$ be a commutative integral domain with quotient field $K$ satisfying:

$\left(^{*}\right.$ If $a, b \in R$ and $a^{2}+b^{2} \neq 0$ then $a^{2}\left(a^{2}+b^{2}\right)^{-1}$ and $a b\left(a^{2}+b^{2}\right)^{-1}$ lie in $R$.

Then $R$ is a Prüfer domain. If $\sqrt{-1} \in K$, then $R=K$.

Since the elements in $\left({ }^{*}\right)$ are homogeneous of degree 0 , it would be equivalent to require $\left(^{*}\right)$ for all $a, b \in X$ with $a^{2}+b^{2} \neq 0$, or to require $\left(1+x^{2}\right)^{-1}, x\left(1+x^{2}\right)^{-1} \in R$ for all $x \neq \pm \sqrt{-1}$ in $K$. 
Proof. If $i=\sqrt{-1} \in K$ then $(1+i x)\left(1+x^{2}\right)^{-1}=(1-i x)^{-1}$ can be anything in $K$ except 0 or $1 / 2$ so $R=K$. Therefore we can assume that $\sqrt{-1} \notin K$. Let $P$ be a prime ideal of $R$. We must show that $R_{P}$ is a valuation ring, i.e. if $u \in K$ then $u$ or $u^{-1}$ lies in $R_{P}$. Since $\sqrt{-1} \notin K$, $1+u^{2} \neq 0$, so $x=\left(1+u^{2}\right)^{-1}$ and $y=u\left(1+u^{2}\right)^{-1}$ lie in $R$ and so does $1-x=u^{2}\left(1+u^{2}\right)^{-1}$. One of $x, 1-x$ is not in $P$ so one of $y x^{-1}=u$, $y(1-x)^{-1}$ lies in $R_{P}$.

Now let $A$ be any commutative domain with quotient field $K$. Define $A^{\#}$ to be the result of adjoining $\left(1+x^{2}\right)^{-1}$ and $x\left(1+x^{2}\right)^{-1}$ to $A$ for all $x \neq \pm \sqrt{-1}$ in $K$. Clearly $A^{\#}$ is the smallest subring of $K$ containing $A$ which satisfies $\left({ }^{*}\right)$ of Lemma 1 . Therefore $A^{\#}$ is a Prüfer domain. The following alternative description of $A^{\#}$ will be useful. Let $E(A)$ be the collection of subrings $B$ of $K$ such that there is a finite sequence $A=B_{0} \subset$ $B_{1} \subset \cdots \subset B_{m}=B$ with $B_{i+1}=B_{i}\left[a_{t}^{2}\left(a_{i}^{2}+b_{i}^{2}\right)^{-1}, a_{i} b_{i}\left(a_{l}^{2}+b_{i}^{2}\right)^{-1}\right]$, where $a_{t}, b_{i} \in B_{l}$ and $a_{t}^{2}+b_{i}^{2} \neq 0$. Then $E(A)$ is filtered by inclusion and $A^{\#}=\cup B$ over all $B \in E(A)$.

REMARK. Lam pointed out that if char $K \neq 2$ it would suffice to adjoin only $\left(1+x^{2}\right)^{-1}$ for all $x \in K$ but then the analogue of Lemma 9 would be false, e.g. for $A=\mathbf{R}[y, z]$ and $x=y z^{-1}$. This follows from Dress' observation [D] that $2 x\left(1+x^{2}\right)^{-1}=\left(y^{2}-z^{2}\right)\left(y^{2}+z^{2}\right)^{-1}$ with $y=x+1$ and $z=x-1$. Another approach is given in [LC, 11.4].

For Theorem 1 we start with $B_{n}=\mathbf{R}\left[x_{0}, x_{1}, \ldots, x_{n}\right] /\left(\sum x_{i}^{2}-1\right)$, let $A_{n}$ be the R-subalgebra of $B_{n}$ generated by all $x_{l} x_{J}$, and take $R=A_{n}^{\#}$. The ideal $I_{n}$ is $\left(x_{0}^{2}, x_{0} x_{1}, \ldots, x_{0} x_{n}\right)$. The ring $A_{n}$ was considered in [SV, Example 2]. It is clearly a domain for $n \geq 1$ since $\Sigma x_{t}^{2}-1$ is irreducible. It is easy to see that $\sqrt{-1}$ does not lie in the quotient field $K$ of $A_{n}$. In fact $K$ is the pure transcendental extension $\mathbf{R}\left(x_{1} x_{0}^{-1}, \ldots, x_{n} x_{0}^{-1}\right)$ since $x_{0}^{-2}=1+\Sigma\left(x_{l} x_{0}^{-1}\right)^{2}$. By [ST, Th. 5.4], $\operatorname{dim} R \leq n$. The reverse inequality will follow from Heitmann's result [H, Th. 3.1] once we show that $\mu\left(I_{n}\right)=n+1$.

For Theorem 2 we start with the tensor product $A$ of all the $A_{n}$ for $n \geq 1$. In other words, let $C=\mathbf{R}\left[x_{l}^{(n)} ; 0 \leq i \leq n, n \geq 1\right]$, let $J \subset C$ be the ideal generated by all $\sum_{0}^{n} x_{i}^{(n) 2}-1$, and let $A \subset B=C / J$ be the R-subalgebra generated by all $x_{i}^{(n)} x_{j}^{(n)}$. Then set $R=A^{\#}$ with $I_{n}=$ $\left(x_{0}^{(n) 2}, x_{0}^{(n)} x_{1}^{(n)}, \ldots, x_{0}^{(n)} x_{n}^{(n)}\right)$. Here also $A$ is a domain whose quotient field $K$ does not contain $\sqrt{-1}$ since $A$ is a subring of $K=R\left(x_{l}^{(n)} x_{0}^{(n)-1} ; 1 \leq i\right.$ $\leq n$ ), a pure transcendental extension of $\mathbf{R}$. Note that $A$ can also be obtained as the union of the subrings $A^{(N)}=A_{1} \otimes_{\mathbf{R}} \cdots \otimes_{\mathbf{R}} A_{N}$. The following observation is useful in this situation. 
LEMma 2. If $A$ is the filtered union of subrings $A_{\alpha}$ then $A^{\#}$ is the filtered union of the $A_{\alpha}^{\#}$ and therefore also of the $B \in \cup E\left(A_{\alpha}\right)$.

Proof. If $A_{\alpha} \subset A_{\beta}$ then $A_{\alpha}^{\#} \subset A_{\beta}^{\#}$. Therefore $\cup A_{\alpha}^{\#}$ is a filtered union and lies in $A^{\#}$. The reverse inclusion follows from the fact that $\cup A_{\alpha}^{\#}$ satisfies $\left({ }^{*}\right)$ of Lemma 1 and $A^{\#}$ is the smallest such ring containing $A$.

2. Real algebraic varieties. We recall here some standard facts of real algebraic geometry. If $A$ is a commutative $\mathbf{R}$-algebra, let $V(A)=$ $\operatorname{Hom}_{\mathbf{R} \text {-alg }}(A, \mathbf{R})$. Each $a \in A$ determines a real valued function $\hat{a}$ on $V(A)$ by the Gelfand representation $\hat{a}(\alpha)=\alpha(a)$. We give $V(A)$ the coarsest topology such that all $\hat{a}$ are continuous. It is clear that $V$ is then a contravariant functor from $\mathbf{R}$-algebras to topological spaces. The Gelfand representation gives a natural $\mathbf{R}$-algebra map $A \rightarrow C(V(A))$, where $C$ denotes the ring of continuous real valued functions. These definitions are identical with those used for Banach algebras $[\mathbf{L}]$ with $\mathbf{R}$ replacing $\mathbf{C}$. However $V(A)$ will usually not be compact if $A$ is not a Banach algebra.

If $A=\mathbf{R}\left[x_{1}, \ldots, x_{n}\right] /\left(f_{1}, \ldots, f_{m}\right)$, all $\hat{a}$ will be continuous if $\hat{x}_{1}, \ldots, \hat{x}_{n}$ are. It follows that $\left(\hat{x}_{1}, \ldots, \hat{x}_{n}\right): V(A) \rightarrow \mathbf{R}^{n}$ gives a homeomorphism of $V(A)$ onto the real algebraic set $\left\{a \in \mathbf{R}^{n} \mid f_{1}(a)=\cdots=f_{m}(a)=0\right\}$.

Recall that Dubois and Efroymson [DE] define a ring $A$ to be (formally) real if $\Sigma a_{i}^{2}=0, a_{i} \in A$, implies $a_{i}=0$ for all $i$.

Lemma 3 [DE]. Let $A$ be of finite type over $\mathbf{R}$. Then $A \rightarrow C(V(A))$ is injective if and only if $A$ is real.

A simple proof is given in [ST, Cor. 10.5c]. Only the special case proved in [ST, Th. 10.4] will be needed here. Some restriction on $A$ is clearly needed, e.g. $V(\mathbf{R}(x))=0$.

Note that if $A$ is a domain with quotient field $K$, then $A$ is real if and only if $K$ is: If $\Sigma a_{i}^{2}=0$ in $K$ we need only clear denominators.

Suppose $X \subset \mathbf{R}^{n}$ is defined by equations $f_{1}=\cdots=f_{m}=0$, where the $f_{i}$ are continuously differentiable functions. For $x \in X$, let $J_{x}$ denote the Jacobian matrix $\left(\partial f_{i} / \partial x_{j}\right)$ at $x$. Let $r=\max \operatorname{rank}\left(J_{x}\right)$ over $x \in X$ and set $X_{\text {reg }}=\left\{x \in X \mid \operatorname{rank}\left(J_{x}\right)=r\right\}$ and $X_{\text {sing }}=\left\{x \in X \mid \operatorname{rank}\left(J_{x}\right)<r\right\}$. The following is a well-known result of Whitney [W].

LEMMA 4. [W]. $X_{\text {reg }}$ is an n-r manifold.

Proof. Suppose $0 \in X_{\mathrm{reg}}$ and the principal $r \times r$ minor of $J_{x}$ is non-zero. By the implicit function theorem, $f_{1}, \ldots, f_{r}, x_{r+1}, \ldots, x_{n}$ are local 
coordinates at 0 . Therefore, in a neighborhood of 0 , we can assume $f_{i}=x_{i}$ for $i \leq r$. Since $\operatorname{rank}\left(J_{x}\right) \leq r$, we see that $\partial f_{i} / \partial x_{j}=0$ for $j>r$, so the $f_{i}$ depend only on $x_{1}, \ldots, x_{r}$, and $X$, near 0 , is given by $x_{1}=\cdots=x_{r}=0$.

The next two lemmas (modulo Lemma 3 ) were proved in [BH] by complexifying.

LEMMA 5. Let $A$ be a d-dimensional domain of finite type over $\mathbf{R}$. Then $\operatorname{dim} V(A) \leq d$ with equality if and only if $A$ is real.

Proof. Let $K$ be the quotient field of A. Then $\mathrm{d}=\operatorname{transc}(K / \mathbf{R})[\mathbf{A K}$, Ch. III, Th. 2.6ii]. Write $\boldsymbol{A}=\mathbf{R}\left[x_{1}, \ldots, x_{n}\right] /\left(f_{1}, \ldots, f_{m}\right)$ and let $\boldsymbol{J}=$ $\left(\partial f_{l} / \partial x_{j}\right)$ as a matrix over $A$. Then $\hat{J}$ is the function $x \mapsto J_{x}$ considered above. By [R, Ch. III, §2, p. 31] $J$ is a presentation matrix for $\Omega_{A / \mathbf{R}}$ as an $A$-module. Therefore, as a matrix over $K$, it presents $K \otimes_{A} \Omega_{A / \mathbf{R}}=\Omega_{K / \mathbf{R}}$ [R, Ch. III, §2, Prop. 4]. This is a vector space of dimension $d$ over $K$ by [ZS, Ch. II, §17, Th. 41] (since $\mathscr{Q}_{K / \mathbf{R}}=\operatorname{Hom}_{K}\left(\Omega_{K / \mathbf{R}}, K\right)$ ). It follows that $\operatorname{rank}(J)=n-d$, so $\operatorname{rank}\left(J_{x}\right) \leq n-d$ for $x \in X$. If $A$ is real, Lemma 3 shows that $\max \operatorname{rank}\left(J_{x}\right)=n-d$ so $\operatorname{dim} V(A)_{\mathrm{reg}}=d$. If $A$ is not real, $V(A)=V(A / I)$, where $I$ is the kernel of $A \rightarrow C(V(A))$. Since $\operatorname{dim} A / I<$ $\operatorname{dim} A$, we can use induction on $d$. If $A$ is real, let $I$ be the ideal of $A$ generated by the $n-d \times n-d$ minors of $J$. Then $V(A)_{\text {sing }}=V(A / I)$. By induction on $d, \operatorname{dim} V(A)_{\text {sing }}<d$. It follows that $\operatorname{dim} V(A)=d$, using either the fact that $V(A)$ is triangulable [ $\mathbf{k}]$, [DK], or by covering $V(A)_{\text {reg }}$ with a countable number of closed $d$-cells and using the sum theorem for dimension [HW].

Lemma 6. Let $A$ be as in lemma 5 and real. Then $\operatorname{dim} V(A)_{\text {sing }}<d=$ $\operatorname{dim} A$. If $A$ is normal, $\operatorname{dim} V(A)_{\text {sing }} \leq d-2$.

Proof. The first statement was proved in the proof of Lemma 5. As in that proof, let $I$ be the ideal of $A$ generated by the $n-d \times n-d$ minors of $J$ so that $V(A)_{\text {sing }}=V(A / I)$. It will suffice to show that $\operatorname{dim} A / I \leq$ $d-2$ or, by [AK, Ch. III, Th. 2.6iii], that no prime ideal of height 1 contains $I$. Suppose there is such a prime ideal $P$. Since $A$ is normal, $A_{P}$ is a discrete valuation ring and $P_{P}=(g)$. If $L=A_{P} / P_{,}$, is the residue field, the matrix $J^{\prime}$, obtained from $J$ by adjoining the row $\left(\partial g / \partial x_{1}, \ldots, \partial g / \partial x_{n}\right)$ and reducing $\bmod P_{P}$, gives a presentation for $\Omega_{L / \mathbf{R}}[\mathbf{R}$, Ch. III, §2, Prop. 7]. Since $P \supset I, \operatorname{rank}(J \bmod P) \leq n-d-1$, so $J^{\prime}$ has $\operatorname{rank} \leq n-d$. Therefore $\operatorname{dim}_{L} \Omega_{L / \mathbf{R}} \geq d$, contradicting the fact that $\operatorname{transc}(L / \mathbf{R})=$ $\operatorname{dim} A / P=d-1$ since $L$ is the quotient field of $A / P$. 
Lemma 7. Let $A$ be a real domain of finite type over $\mathbf{R}$. Let $a \in A$ be non-zero. Then $\left\{x \in V(A)_{\mathrm{reg}} \mid \hat{a}(x) \neq 0\right\}$ is dense in $V(A)_{\mathrm{reg}}$.

Note that $\{x \in V(A) \mid \hat{a}(x) \neq 0\}=V\left(A_{a}\right)$, so the set in question is $V(A)_{\mathrm{reg}} \cap V\left(A_{a}\right)$. It follows that $V(A)_{\mathrm{reg}} \cap V\left(A_{a}\right) \neq \varnothing$. The non-connected curve $y^{2}=x^{2}(x-1)[\mathbf{M}, \mathrm{p} .12]$ with $a=x$ shows that $V\left(A_{a}\right)$ need not be dense in $V(A)$.

Proof. Clearly $V(A)=V\left(A_{a}\right) \cup V(A /(a))$ and $\operatorname{dim} V(A /(a)) \leq$ $\operatorname{dim} A /(a)<\operatorname{dim} A=\operatorname{dim} V(A)=d$. If the conclusion is false, there is a non-empty open set $U$ of $V(A)_{\text {reg }}$ with $U \cap V\left(A_{a}\right)=0$. Therefore, $U \subset$ $V(A /(a))$, but this is impossible since $U$ is a $d$-manifold.

The following result is a classical fact.

Lemma 8. If $B \supset A$ is finite over $A$ then $V(B) \underset{p}{\rightarrow} V(A)$ is proper.

In other words, $p^{-1}(C)$ is compact if $C$ is. In particular, $V(B)$ is compact if $V(A)$ is.

Proof. It is enough to look at the case $B=A[x]$ where $x \in B$ satisfies $x^{n}+a_{1} x^{n-1}+\cdots+a_{n}=0, a_{i} \in A$. Let $C \subset V(A)$ be compact. Since the $\hat{a}_{l}$ are bounded on $C, \hat{x}$ is bounded on $p^{-1}(C)$, so $p^{-1}(C)$ is a closed subset of $C \times\{x \in \mathbf{R} \| x \mid \leq M\}$ for some large $M<\infty$.

A similar result holds for the construction used in $\$ 1$.

Lemma 9. Let $A$ be a domain with quotient field $K$. Let $a, b \in A$ with $a^{2}+b^{2} \neq 0$. Let $x=a^{2}\left(a^{2}+b^{2}\right)^{-1}, y=a b\left(a^{2}+b^{2}\right)^{-1}$, and $B=A[x, y]$ $\subset K$. Then $V(B) \rightarrow V(A)$ is proper. Therefore, $V(B) \rightarrow V(A)$ is proper for $B \in E(A)$.

Proof. Since $(x-1 / 2)^{2}+y^{2}=1 / 4, V(B)$ is a closed subset of $V(A) \times S$, where $S$ is the circle defined by this equation.

3. Homological properties. Throughout this paper, $H^{i}$ denotes Čech cohomology with coefficient group $\mathbf{Z} / 2 \mathbf{Z}$.

Recall that a relative $n$-manifold is a compact pair $(X, A)$ such that $X-A$ is an $n$-manifold.

Lemma 10. Let $(X, A)$ be a relative $n$-manifold and let $U \subset X-A$ be an open $n$-cell. Then $H^{n}(X, X-U) \rightarrow H^{n}(X, A)$ is injective. If $X-A$ is connected, this map is an isomorphism. 
This is an immediate consequence of Poincaré-Lefschetz duality. An elementary proof for $X-A$ connected is given in [ES, Chapter XI, Theorem 6.8iv]. If $X-A$ has components $U_{\alpha}$, then $H^{n}(X, A)=$ $\bigoplus H^{n}\left(X, X-U_{\alpha}\right)$ by [ES, Chapter X, Exercise B3] (cf. [ES, Chapter XI, Theorem 6.10]) and the required result follows.

The following lemma uses an argument from [BH].

LemMa 11. Let $A$ be a real normal domain of finite type over $\mathbf{R}$ with $\operatorname{dim} A=n$. Assume $X=V(A)$ is compact. Let $U \subset X$ be an $n$-cell. Then $H^{n}(X, X-U) \rightarrow H^{n}(X)$ is injective.

Proof. Since $\operatorname{dim} X_{\text {sing }}<\underset{\approx}{\sim} U$ meets $X_{\text {reg }}$. Let $W \subset X_{\text {reg }} \cap U$ be an $n$-cell. Then $H^{n}(X, X-W) \stackrel{\approx}{\rightarrow} H^{n}(X, X-U)$ by Lemma 10 , so it is enough to prove the lemma with $U$ replaced by $W$. Since $\left(X, X_{\text {sing }}\right)$ is a relative $n$-manifold by Lemmas 4 and $5, H^{n}(X, X-W) \rightarrow H^{n}\left(X, X_{\text {sing }}\right)$ is injective by Lemma 10 . Finally, since $\operatorname{dim} X_{\text {sing }} \leq n-2$ by Lemma 6 , the exact cohomology sequence of $\left(X, X_{\text {sing }}\right)$ shows that $H^{n}\left(X, X_{\text {sing }}\right) \stackrel{\approx}{\rightarrow} H^{n}(X)$.

LEMMA 12. Let $A$ be a real domain of finite type over $\mathbf{R}$ with quotient field $K$. Let $A \subset B \subset K$ with $B$ also of finite type over $\mathbf{R}$. Suppose $V(B)$ is compact and $V(A)$ is a compact connected manifold. Then $p: V(B) \rightarrow V(A)$ induces an injective map of cohomology $H^{\prime}(V(A)) \rightarrow H^{i}(V(B))$ for all $i$.

Proof. Let $B^{\prime}$ be the integral closure of $B$ in $K$. It is finite over $B$ and so of finite type over $\mathbf{R}$ [ZS, Chapter $\mathrm{V}, \S 4$, Theorem 9], and $V\left(B^{\prime}\right)$ is compact by Lemma 8 . By replacing $B$ by $B^{\prime}$, we can assume $B$ is normal. This idea is also taken from $[\mathbf{B H}]$. Note that $B$ is real since $A$, and therefore $K$, is. Also $\operatorname{dim} B=\operatorname{transc}(K / \mathbf{R})=\operatorname{dim} A=n$, say. Let $a \in A$ be a common denominator for a finite set of generators of $B$ expressed as fractions from $A$. Then $A_{a}=B_{a}$ so $p: V\left(B_{a}\right) \cong V\left(A_{a}\right)$. By Lemma 7 we can find an $n$-cell $U \subset V\left(B_{a}\right) \cap V(B)_{\text {reg }}$. Clearly $p: U \cong W=p(U) \subset$ $V\left(A_{a}\right)$. Consider the diagram

$$
\begin{array}{ccc}
H^{n}(V(B), V(B)-U) & \stackrel{\beta}{\rightarrow} & H^{n}(V(B)) \\
\gamma \uparrow \approx & & \uparrow p^{*} \\
H^{n}(V(A), V(A)-W) & \stackrel{\alpha}{\rightarrow} & H^{n}(V(A)) .
\end{array}
$$

Here $\gamma$ is an isomorphism by excision [ES, Ch. X, Th. 5.4], $\alpha$ is an isomorphism by Lemma 10 , and $\beta$ is injective by Lemma 11 . Therefore, 
$p^{*}: H^{n}(V(A)) \rightarrow H^{n}(V(B))$ is injective. We extend this to $H^{l}$ by a trick of Hopf [Ho]. Let $u \in H^{i}(V(A))$ be non-zero. By Poincare duality, there is some $v \in H^{n-i}(V(A))$ with $u v \neq 0$ in $H^{n}(V(A))$. Therefore, $0 \neq p^{*}(u v)$ $=p^{*}(u) p^{*}(v)$, so $p^{*}(u) \neq 0$.

REMARK. In the application given here, the use of Poincare duality can be avoided. For Theorem 1, only $H^{n}$ is needed. For Theorem 2, $V(A)$ will be a product of two manifolds $M^{p} \times N^{q}$ and we will need $p^{*}(u) \neq 0$ only for an element $u$ of the form $u=\operatorname{pr}_{1}^{*}\left(u^{\prime}\right)$, where $u^{\prime} \in H^{p}(M)$ and $\operatorname{pr}_{1}$ is the projection on $M$. If $v^{\prime} \in H^{q}(N)$ is non-zero, then $u \operatorname{pr}_{2}^{*}\left(v^{\prime}\right)=u^{\prime} \times$ $v^{\prime} \neq 0$ by the Künneth formula and the above argument applies.

REMARK. Under the hypotheses of Lemma 12, it follows that $V(B) \rightarrow$ $V(A)$ is onto. This can be generalized as follows: If $A \subset B$ are domains of finite type over $\mathbf{R}$ with the same quotient field $K$ and if $p: V(B) \rightarrow V(A)$ is proper (e.g. if $B \in E(A)$ ), then $V(A)_{\text {reg }} \subset p(V(B))$ ). This follows from Lemma 7 and the observation above that $V\left(B_{a}\right) \stackrel{\cong}{\rightrightarrows} V\left(A_{a}\right)$, since a proper map of locally compact spaces is closed. The non-connected curve $A=$ $\mathbf{R}[x, y] /\left(y^{2}-x^{2}(x-1)\right)$ with $B=A\left[x^{2}\left(x^{2}+y^{2}\right)^{-1}, \quad x y\left(x^{2}+y^{2}\right)^{-1}\right]$ shows that $p$ need not be onto. Here $x^{2}\left(x^{2}+y^{2}\right)^{-1}=x^{-1}$, so the isolated point is not in $p(V(B))$.

4. Proof of the theorems. In [SV] $I$ showed that for certain classes of spaces $X$, in particular the compact ones, the section functor gives an equivalence between the category of vector bundles on $X$ and the category of finitely generated projective modules over the ring of continuous functions $C(X)$. It is easy to check that this is natural: If $f: X \rightarrow Y$ and $\xi$ is a vector bundle on $Y$, we get a map $\Gamma(\xi) \rightarrow \Gamma\left(f^{*} \xi\right)$ taking a section $s$ to $s \circ f$ which can be interpreted as a section of $f^{*} \xi$ since we have a cartesian diagram

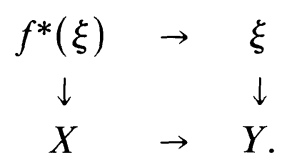

This map is semilinear with respect to $C(Y) \rightarrow C(X)$ and so induces a $\operatorname{map} \theta_{\xi}: C(X) \otimes_{C(Y)} \Gamma(\xi) \rightarrow \Gamma\left(f^{*} \xi\right)$.

LEMMA 13. If $\xi$ is a direct summand of a trivial bundle, $\theta_{\xi}$ is an isomorphism.

The hypothesis is satisfied for the spaces considered in [SV]. 
Proof. Clearly $\theta_{\xi \oplus \eta}=\theta_{\xi} \oplus \theta_{\eta}$. Using this we reduce to the case where $\xi$ is trivial. The result is obvious in this case.

Now let $A_{n}$ be as in $\$ 1$. In [SV, Example 2] it was observed that there is a map $A_{n} \rightarrow C\left(\mathbf{P}^{n}\right)$, where $\mathbf{P}^{n}$ is real projective $n$-space. We need only regard $\mathbf{P}^{n}$ as the quotient of $S^{n}$ by the antipodal identification and send the $x_{i}$ to the usual coordinate functions. It is very easy to check that $\mathbf{P}^{n}=V\left(A_{n}\right)$ by using the relations $\left(x_{i} x_{j}\right)\left(x_{p} x_{q}\right)=\left(x_{i} x_{p}\right)\left(x_{j} x_{q}\right)$ and $\Sigma x_{i}^{2}$ $=1$. Since $\operatorname{dim} A_{n}=n=\operatorname{dim} \mathbf{P}^{n}$, Lemma 5 shows that $A_{n}$ is real and $A_{n} \rightarrow C\left(\mathbf{P}^{n}\right)$ is injective. It is also quite easy to check this directly. From [SV, Example 2] we see that the invertible ideal $I=\left(x_{0}^{2}, x_{0} x_{1}, \ldots, x_{0} x_{n}\right)$ of $A_{n}$ corresponds to the canonical line bundle $\gamma$ on $\mathbf{P}^{n}$, i.e. $C\left(\mathbf{P}^{n}\right) \otimes_{A_{n}} I \approx$ $\Gamma(\gamma)$. The total Stiefel-Whitney class of $\gamma$ is $w(\gamma)=1+\alpha$, where $\alpha$ generates $H^{1}\left(\mathbf{P}^{n}\right)=\mathbf{Z} / 2 \mathbf{Z}$. It is a unit in $H^{*}\left(\mathbf{P}^{n}\right)=\mathbf{Z} / 2 \mathbf{Z}[\alpha] /\left(\alpha^{n+1}\right)$ with inverse $w(\gamma)^{-1}=1+\alpha+\cdots+\alpha^{n}[\mathbf{M S}]$.

Form $R=A_{n}^{\#}$ as in $\S 1$ and suppose $I_{n}=R I$ requires fewer than $n+1$ generators, say $R I=\left(y_{1}, \ldots, y_{k}\right)$ with $k \leq n$. Write $y_{i}=\Sigma a_{i j} x_{0} x_{j}$ and $x_{0} x_{j}=\sum b_{j l} y_{l}$ with $a_{i j}, b_{j i} \in R$. Some $B \in E\left(A_{n}\right)$ contains all $a_{\imath \jmath}$ and $b_{j l}$ so $B I=\left(y_{1}, \ldots, y_{k}\right) B$ has $k$ generators. ${ }^{2}$ Note that $B \otimes_{A_{n}} I \stackrel{\approx}{\rightarrow} B I$ since $I$ is locally principal. By Lemma $13, B I$ corresponds to the line bundle $p^{*}(\gamma)$ on $V(B)$ where $p: V(B) \rightarrow V(A)$. We can find $0 \rightarrow M \rightarrow B^{k} \rightarrow B I \rightarrow 0$. This splits since $I$ is invertible so $B^{k} \approx M \oplus B I$. Therefore, $o^{k} \approx \mu \oplus p^{*}(\gamma)$, where $o$ is the trivial line bundle and $\mu$ corresponds to $M$. Now $w(\mu) w\left(p^{*} \gamma\right)=w\left(o^{k}\right)=1$, so $w(\mu)=w\left(p^{*} \gamma\right)^{-1}=p^{*}\left(w(\gamma)^{-1}\right)$. Therefore, $w_{n}(\mu)=p^{*}\left(\alpha^{n}\right) \neq 0$ by Lemma 12. But $\mu$ has rank $k-1$ so $w_{i}(\mu)=0$ for $i \geq k$ [MS]. The assumption that $k \leq n$ thus leads to a contradiction.

For Theorem 2 we use the same method. If $I_{n}$ has $k \leq n$ generators, the same will be true for $\left(x_{0}^{(n) 2}, \ldots, x_{0}^{(n)} x(n)_{n}\right) B$ for some $B \in E\left(A^{(N)}\right)$ with $A^{(N)}$ as in §1. This follows from Lemma 2. Now $V\left(A^{(N)}\right)=\Pi_{1}^{N} \mathbf{P}^{r}$ and $\left(x_{0}^{(n) 2}, \ldots, x_{0}^{(n)} x_{n}^{(n)}\right) A^{(N)}$ corresponds to the bundle $\operatorname{pr}_{n}^{*}(\gamma)$, where $\gamma$ is as above and $\operatorname{pr}_{n}$ is the projection of $V\left(A^{(N)}\right)$ on $\mathbf{P}^{n}$. Since $\mathrm{pr}_{n}$ has a section, $\operatorname{pr}_{n}^{*}: H^{*}\left(\mathbf{P}^{n}\right) \rightarrow H^{*}\left(V\left(A^{(N)}\right)\right)$ is injective. As above we find $p^{*} \operatorname{pr}_{n}^{*}(\gamma) \oplus \mu \approx o^{k}$, getting $w_{n}(\mu)=p^{*} \operatorname{pr}_{n}^{*}\left(\alpha^{n}\right) \neq 0$ by Lemma 12. This gives the same contradiction as before.

5. Further results. In $[\mathbf{H}]$ Heitmann proves the following results about $n$-dimensional Prüfer domains $R$.

(1) Finitely generated ideals $I$ have $\mu(I) \leq n+1$.

\footnotetext{
${ }^{2}$ This step, and similar arguments below, can be avoided by using the continuity property of Čech cohomology to extend Lemma 12 to the case $B=A^{\#}$.
} 
(2) If $M$ is a finitely generated torsion-free module of rank $d$ then $\mu(M) \leq n+d$.

(3) If $M$ is as in (2) and $d>n$ then $M$ has a free summand.

(4) The stable range of $R$ is $\operatorname{sr}(R)=n+1$.

I will show that each of the results is the best possible. This is clear for (1) by Theorems 1 and 2 .

THEOREM 3. Let $I_{n}$ be as in Theorem 1 or 2 . Let $d \geq 1$ be any integer and set $M=I_{n} \oplus R^{d-1}$. Then $\mu(M)=n+d$.

Proof. It is trivial that $\mu(M) \leq n+d$. The converse is proved by the same method as in $\$ 4$ since adding on free summands does not affect the Stiefel-Whitney classes (cf. [SV, Example 2]), [MS].

THEOREM 4. Let $I_{n}$ be as in Theorem 1 or 2. Let $M$ be the kernel of any epimorphism $R^{n+1} \rightarrow I_{n}$. Then $M$ is finitely generated projective of rank $n$ with no non-trivial free summand.

Proof. Since the epimorphism splits, cancellation [KA, p. 75] shows that $M$ is unique up to isomorphism. Therefore we can restrict our attention to the obvious epimorphism sending the standard base of $R^{n+1}$ to the generators of $I_{n}$ given in $\S 1$. Let $M_{0}$ be defined like $M$ using $A_{n}$ or $A^{(n)}$ in place of $R$. Then $M=R M_{0}$. If $M$ had a free summand, we could find $B \in E\left(A^{\prime}\right), A^{\prime}=A_{n}$ or $A^{(k)}$ with $B M_{0}=B \oplus N$. Let $p: V(B) \rightarrow V\left(A^{\prime}\right)$ and let $A^{\prime} M_{0}$ and $N$ correspond to the bundles $\mu$ and $\nu$ on $V\left(A^{\prime}\right)$ and $V(B)$. Then $p^{*}(\mu)=o \oplus \nu$, so $w_{n}\left(p^{*} \mu\right)=w_{n}(\nu)=0$ since $\nu$ has rank $n-1$. This gives the same contradiction as in $\$ 4$.

THEOREM 5. For any integer $n \geq 1$, there is a Prüfer domain $R$ with $\operatorname{dim} R=n$ and $\operatorname{sr}(R)=n+1$.

Proof. The proof was suggested by Vaserštein's proof that $\operatorname{sr} \mathbf{R}\left[x_{1}, \ldots, x_{n}\right]=n+1[\mathbf{V}$, Th. 8]. We start with

$$
B_{n}=\mathbf{R}\left[x_{0}, \ldots, x_{n}\right] /\left(\sum x_{i}^{2}-1\right)
$$

and apply the construction of $\S 1$ to get $R=B_{n}^{\#}$. We have $\operatorname{dim} R \leq n$, and the reverse inequality will follow from (4) once we show that $\operatorname{sr}(R) \geq n+$ 1. Consider the unimodular row $\left(x_{0}, \ldots, x_{n}\right)$ over $R$. I claim there are no $a_{i} \in R$ such that $\left(x_{0}+a_{0} x_{n}, \ldots, x_{n-1}+a_{n-1} x_{n}\right)$ is unimodular. If there were such $a_{l}$, they and the elements $b_{i}$ needed to write $\Sigma b_{i}\left(x_{l}+a_{i} x_{n}\right)=1$ 
would lie in some $B \in E\left(A_{n}\right)$. Let $X=V(B), I=[0,1]$, and define $f$ : $X \times I \rightarrow R^{n+1}$ by

$$
\begin{array}{r}
f(x, t)=\left(\hat{x}_{0}(x)+t \hat{a}_{0}(x) \hat{x}_{n}(x), \ldots, \hat{x}_{n-1}(x)+t \hat{a}_{n-1}(x) \hat{x}_{n}(x),\right. \\
\left.(1-t) \hat{x}_{n}(x)\right) .
\end{array}
$$

The unimodularity assumptions show that $f(x, t)$ is never 0 , so we can define $g: X \times I \rightarrow S^{n}$ by $g(x, t)=f(x, t) /\|f(x, t)\|$. Now $g(-, 0)$ is the map $p: X=V(B) \rightarrow S^{n}=V\left(A_{n}\right)$ induced by $A_{n} \subset B$, while $g(-, 1)=q$ maps $X$ into $S^{n-1} \subset S^{n}$ and so is nullhomotopic. This gives the required contradiction since $p^{*}: H^{n}\left(S^{n}\right) \rightarrow H^{n}(X)$ is non-trivial by Lemma 12 , while $p^{*}=q^{*}=0$.

\section{THeOREM 6. There is a Prüfer domain $R$ with $\operatorname{sr}(R)=\infty$.}

Proof. Let $B$ be the tensor product of all the rings $B_{n}$ considered above for $n \geq 1$. Then $B=\cup B^{(n)}$ with $B^{(n)}=B_{1} \otimes B_{2} \otimes \cdots \otimes B_{n}$. These are all domains whose quotient field does not contain $\sqrt{-1}$, e.g. since $\mathbf{C} \otimes_{\mathbf{R}} B_{n}$ is a domain with quotient field $\mathbf{C}\left(x_{0}, \ldots, x_{n-2}, x_{n-1}+\sqrt{-1} x_{n}\right)$. Since $V\left(B^{(n)}\right)=S^{1} \times \cdots \times S^{n}$, Lemma 5 shows that $B^{(n)}$ is real. Let $R=B^{\sharp}$. If $\operatorname{sr}(R) \leq n$, take the unimodular row $\left(x_{0}^{(n)}, \ldots, x_{n}^{(n)}\right)$, where, as before, $x_{i}^{(n)}$ is the image of $x_{i} \in B_{n}$ in $B$. As in the proof of Theorem 5 we can find $C \in E\left(B^{(N)}\right)$ and $a_{i} \in C$ such that $\left(x_{0}^{(n)}+a_{0} x_{n}^{(n)}, \ldots, x_{n-1}^{(n)}+\right.$ $\left.a_{n-1} x_{n}^{(n)}\right)$ is unimodular over $C$. As above we see that $\operatorname{pr}_{n} p: V(C) \rightarrow$ $V\left(B^{(N)}\right) \rightarrow S^{n}$ is nullhomotopic, contradicting the fact that $\left(\operatorname{pr}_{n} p\right)^{*}$ : $H^{n}\left(S^{n}\right) \rightarrow H^{n}(V(C))$ is injective by Lemma 12 .

6. Relation to Schülting's work. The construction of $\S 1$ can be modified as in [S] by adjoining elements of the form $x_{i j}=a_{i} a_{j}\left(\Sigma a_{l}^{2}\right)^{-1}$ for $a_{i} \in A$ (or even in $K$ ) with $\Sigma a_{i}^{2} \neq 0$. This leads to a Prüfer domain $A^{\%} \supset A^{\#}$ which can replace $A^{\#}$ in all the above theorems. We need only verify the analogue of Lemma 9 which follows from the identity $\sum\left(2 x_{i i}-1\right)^{2}+4 \sum_{i \neq j} x_{i j}^{2}=n$.

If these constructions are applied to the prime subring $\mathbf{Z} 1 \subset K$ (using all $a, b$, resp. $a_{i}$, in $\left.K\right)$ we get a ring $D_{K}=(\mathbf{Z} 1)^{\#}$ which occurs in [D] and the ring $A_{K}=(\mathbf{Z} 1)^{\%}$ defined in [S]. It is clear that for any $A \subset K$ we have $A^{\%}=A D_{K}$ and $A^{\%}=A A_{K}$. Dress [D] showed that $D_{K}$ is a Prüfer domain using the argument of Lemma 1 . If $K$ is formally real, Schülting showed that $A_{K}$ is the intersection of all valuation rings $V$ of $K$ whose residue fields $V / M$ are formally real. He defined $A_{K}$ by adjoining only elements 
of the form $\left(1+\sum x_{i}^{2}\right)^{-1}$. However, the same result is true for the definition of $A_{K}$ used here. If $V / M$ is formally real, let $\left|a_{1}\right|$ be maximal for the valuation corresponding to $V$. Dividing the numerator and denominator of $x_{i j}$ by $a_{1}^{2}$ shows that $\left|x_{i j}\right| \leq 1$ so $x_{i j} \in V$. It follows that the present definition of $A_{K}$ agrees with Schülting's. The same argument shows that if $\sqrt{-1} \notin K$ then $D_{K}$ is the intersection of all valuation rings $V$ of $K$ such that $\sqrt{-1} \notin V / M[\mathbf{D}, \S 4]$. It follows that similar characterizations hold for $A^{\%}$ and $A^{\#}$. We have only to add the restriction that $A \subset V$. As in Lemma 1 we will have $A^{\%}=A_{K}=K$ if $K$ is not formally real, since for large $n, Q=x_{1}^{2}+\cdots+x_{n}^{2}$ will be isotropic and $(1+Q)^{-1}$ will represent all elements of $K^{*}$ by [LQ, I, Th. 3.4(3)].

It is now easy to see that the ring $R$ of Theorem 1 for $n=2$ is the same as the one considered by Schülting [S] provided we use the $A^{\%}$ construction. This was pointed out by Gilmer, Heitmann, and Lam. In fact, the ring $A_{n}^{\%}$ was conjectured to have the property of Theorem 1 a few years ago by Gilmer (unpublished) and by Lam [LO, p. 122]. Let $K$ be the quotient field of $A_{n}$. As we saw in $\S 1, K=\mathbf{R}\left(y_{1}, \ldots, y_{n}\right)$, where $y_{i}=x_{t} x_{0}^{-1}$. But $x_{i} x_{j}=y_{i} y_{j}\left(\sum_{0}^{n} y_{i}^{2}\right)^{-1} \in A_{K}$ (with $y_{0}=1$ ), so $A_{n} \subset A_{K}$ and therefore $A_{n}^{\%}=A_{K}$. The ideal used by Schülting is $\left(1, y_{1}, y_{2}\right)=x_{0}^{-2} I_{2}$. Note that $y_{1}, \ldots, y_{n} \notin A_{K}$, otherwise we could find $C \in E\left(A_{n}\right)$ with $A_{n} \subset B=$ $\mathbf{R}\left[y_{1}, \ldots, y_{n},\left(1+\sum_{1}^{n} y_{i}^{2}\right)^{-1}\right] \subset C$. By Lemma $12, H^{n}\left(V\left(A_{n}\right)\right) \rightarrow H^{n}(V(B))$ $\rightarrow H^{n}(V(C))$ is non-trivial, but $H^{n}(V(B))=0$ since $V(B)=\mathbf{R}^{n}$. Since $A_{K}$ is stable under $\operatorname{Aut}(K / R)$, it follows that $A_{K}$ does not contain any set of algebraically independent generators for $K$ over $R$, or even a single element of such a set. In the case of the ring $B_{n}$ used in proving Theorem 5 we also have $B_{n}^{\%}=A_{L}$, where $L$ is the quotient field of $B_{n}$. This is clear from the fact that $\frac{1}{2} x_{i}=1 x_{i}\left(1+\sum x_{j}^{2}\right)^{-1}$.

In contrast to the above results, $A_{n}^{\#} \neq D_{K}$ since $A_{n}$ is not contained in $D_{K}$ for $n \geq 2$. To see this, let $V$ be the discrete valuation ring $\mathbf{R}\left[y_{1}, \ldots, y_{n}\right]_{(f)}$, where $f=1+\sum_{1}^{n} y_{i}^{2}=x_{0}^{-2}$. Then $A_{n} \leftarrow V$, since $x_{0}^{2} \in A_{n}$, while $x_{0}^{-2}=f \in M=f V$. However $\sqrt{-1} \notin V / M$, so $D_{K} \subset V$ : Suppose $g$ and $h$ are polynomials in the $y_{\imath}$ with $g^{2}+h^{2} \equiv 0 \bmod f$. Since $f$ is absolutely irreducible for $n \geq 2$, we can work over $\mathbf{C}$ and get $g \pm i h \equiv 0$ $\bmod f$, which implies $g \equiv h \equiv 0 \bmod f$.

\section{REFERENCES}

[AK] A. Altman and S. Kleiman, Introduction to Grothendieck Duality Theory, Lect. Notes in Math. 146, Springer-Verlag, Berlin 1970

[BH] A. Borel et A. Haefliger, La classe d'homologie fondamentale d'un espace analytique, Bull. Soc. Math. France, 89 (1961), 461-513. 
[CE] H. Cartan and S. Eilenberg, Homological Algebra, Princeton 1956.

[D] A. Dress, Lotschnittebenen mit halbierbarem rechten Winkel, Arch. Math., 16 (1965), 388-392.

[DE] D. W. Dubois and G. Efroymson, Algebraic theory of real varieties I, Studies and Essays presented to $\mathrm{Yu}$-Why Chen on his 60th birthday, Math. Res. Center, Nat. Taiwan Univ., Taipei 1970.

[DK] H. Delfs and M. Knebusch, On the homology of algebraic varieties over real closed fields, to appear.

[ES] S. Eilenberg and N. E. Steenrod, Foundations of Algebraic Topology, Princeton 1952.

[G] R. Gilmer, Multiplicative Ideal Theory, Marcel Dekker, New York 1972.

[G2] _ Two constructions of Prüfer domains, J. Reine Angew. Math., 239/240 (1969), 153-162.

[GG] A note on generating sets for invertible ideals, Proc. Amer. Math. Soc., 22 (1969), 426-427.

[GH] R. Gilmer and W. Heinzer, On the number of generators of an invertible ideal, J. Algebra, 14 (1970), 139-151.

[H] R. C. Heitmann, Generating ideals in Prüfer domains, Pacific J. Math., 62 (1976), 117-126.

[Ho] H. Hopf, Zur Algebra der Abbildungen von Mannigfaltigkeiten, J. Reine Angew. Math., 163 (1930), 71-88.

[HW] W. Hurewicz and H. Wallman, Dimension Theory, Princeton 1948.

[KA] I. Kaplansky, Infinite Abelian Groups, rev. ed., Univ. of Michigan Press, Ann Arbor 1969.

[KC] _ Commutative Rings, Allyn and Bacon, Boston 1970.

[L] L. H. Loomis, An Introduction to Abstract Harmonic Analysis, Van Nostrand, New York 1953.

[k] S. kojasiewicz, Triangulation of semi-analytic sets, Ann. Scuola Norm. Sup. Pisa, (3) 18 (1964), 449-474.

[LC] T-Y. Lam, Ordering, Valuations, and Quadratic Forms, CBMS Notes, Amer. Math. Soc., to appear.

[LO] _ The Theory of Ordered Fields, in Ring Theory and Algebra III, ed. B. R. McDonald, Marcel Dekker, New York 1980.

[LQ] _ _ Algebraic Theory of Quadratic Forms, W. A. Benjamin, Reading, MA 1973

[M] J. Milnor, Singular points of complex hypersurfaces, Ann. of Math. Studies 61, Princeton 1968.

[MS] J. Milnor and J. D. Stasheff, Characteristic Classes, Ann. of Math. Studies 76, Princeton 1974.

[R] M. Raynaud, Anneaux locaux henseliens, Lect. Notes in Math. 169, SpringerVerlag, Berlin 1970.

[S] H-W. Schülting, Uber die Erzeugendenanzahl invertierbarer Ideale in Prüferringen, Comm. Algebra, 7 (1979), 1331-1349.

[ST] R. G. Swan, Topological examples of projective modules, Trans. Amer. Math. Soc., 230 (1977), 201-234.

[SV] _ Vector bundles and projective modules, Trans. Amer. Math. Soc., 105 (1962), 264-277.

[V] L. N. Vaserštein, Stable rank of rings and dimensionality of topological spaces, Funk. An. i Prilozen. 5 (1971), 17-27 transl. Functional Anal. Appl., 5 (1971), 102-110. 
[W] H. Whitney, Elementary structure of real algebraic varieties, Ann. of Math., (2) 66 (1957), 545-556.

[ZS] O. Zariski and P. Samuel, Commutative Algebra vol. I, Van Nostrand, New York 1958.

Received April 12, 1982.

UNIVERSITY OF CHICAGO

Chicago, IL 60637 


\title{
PACIFIC JOURNAL OF MATHEMATICS EDITORS
}

\author{
Donald BABBITT (Managing Editor) \\ University of California \\ Los Angeles, CA 90024 \\ Hugo RossI \\ University of Utah \\ Salt Lake City, UT 84112 \\ C. C. MOore and Arthur Ogus \\ University of California \\ Berkeley, CA 94720
}

J. DUGUNDJI
Department of Mathematics

University of Southern California

Los Angeles, CA 90089-1113

R. FinN and H. SAMELSON

Stanford University

Stanford, CA 94305

\section{ASSOCIATE EDITORS}
R. ARENS
E. F. BECKENBACH
B. H. NeumanN
F. WOLF
K. YosHIDA (1906-1982)

\section{SUPPORTING INSTITUTIONS}

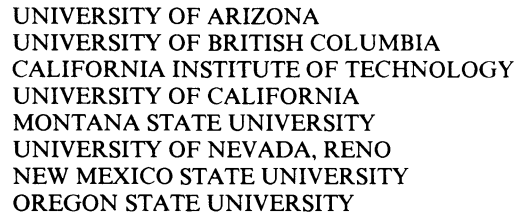

UNIVERSITY OF ARIZONA

UNIVERSITY OF BRITISH COLUMBIA

CALIFORNIA INSTITUTE OF TECHNOLOGY

UNIVERSITY OF CALIFORNIA

MONTANA STATE UNIVERSITY

UNIVERSITY OF NEVADA, RENO

NEW MEXICO STATE UNIVERSITY

OREGON STATE UNIVERSITY

\author{
UNIVERSITY OF OREGON \\ UNIVERSITY OF SOUTHERN CALIFORNIA \\ STANFORD UNIVERSITY \\ UNIVERSITY OF HAWAII \\ UNIVERSITY OF TOKYO \\ UNIVERSITY OF UTAH \\ WASHINGTON STATE UNIVERSITY \\ UNIVERSITY OF WASHINGTON
}

The Supporting Institutions listed above contribute to the cost of publication of this Journal, but they are not owners or publishers and have no responsibility for its content or policies.

Mathematical papers intended for publication in the Pacific Journal of Mathematics should be in typed form or offset-reproduced (not dittoed), double spaced with large margins. Please do not use built up fractions in the text of the manuscript. However, you may use them in the displayed equations. Underline Greek letters in red, German in green, and script in blue. The first paragraph must be capable of being used separately as a synopsis of the entire paper. In particular it should contain no bibliographic references. Please propose a heading for the odd numbered pages of less than 35 characters. Manuscripts, in triplicate, may be sent to any one of the editors. Please classify according to the scheme of Math. Reviews, Index to Vol. 39. Supply name and address of author to whom proofs should be sent. All other communications should be addressed to the managing editor, or Elaine Barth, University of California, Los Angeles, California 90024.

There are page-charges associated with articles appearing in the Pacific Journal of Mathematics. These charges are expected to be paid by the author's University, Government Agency or Company. If the author or authors do not have access to such Institutional support these charges are waived. Single authors will receive 50 free reprints; joint authors will receive a total of 100 free reprints. Additional copies may be obtained at cost in multiples of 50 .

The Pacific Journal of Mathematics is issued monthly as of January 1966. Regular subscription rate: $\$ 132.00$ a year (6 Vol., 12 issues). Special rate: $\$ 66.00$ a year to individual members of supporting institutions.

Subscriptions, orders for numbers issued in the last three calendar years, and changes of address should be sent to Pacific Journal of Mathematics, P.O. Box 969, Carmel Valley, CA 93924, U.S.A. Old back numbers obtainable from Kraus Periodicals Co., Route 100, Millwood, NY 10546.

The Pacific Journal of Mathematics ISSN 0030-8730 is published monthly by the Pacific Journal of Mathematics at P.O. Box 969, Carmel Valley, CA 93924. Application to mail at Second-class postage rates is pending at Carmel Valley, California, and additional mailing offices. Postmaster: Send address changes to Pacific Journal of Mathematics, P. O. Box 969, Carmel Valley, CA 93924.

PUBLISHED BY PACIFIC JOURNAL OF MATHEMATICS, A NON-PROFIT CORPORATION 


\section{Pacific Journal of Mathematics}

\section{Vol. 111, No. $2 \quad$ December, 1984}

Berndt Brenken, Representations and automorphisms of the irrational

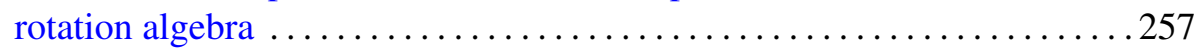

Harold George Diamond, A number theoretic series of I. Kasara . . . . . . 283

Rolf Farnsteiner, On the structure of simple-semiabelian Lie algebras . . . . 287

Guillermo Grabinsky, Poisson process over $\sigma$-finite Markov chains . ......301

Derbiau Frank Hsu and A. Donald Keedwell, Generalized complete

mappings, neofields, sequenceable groups and block designs. I . . . . . 317

William H. Julian and Fred Richman, A uniformly continuous function on

$[0,1]$ that is everywhere different from its infimum $\ldots \ldots \ldots \ldots . \ldots 33$

D. H. Lehmer and Emma Lehmer, The sextic period polynomial .........341

E. Maluta, Uniformly normal structure and related coefficients ...........357

Coy Lewis May, The species of bordered Klein surfaces with maximal

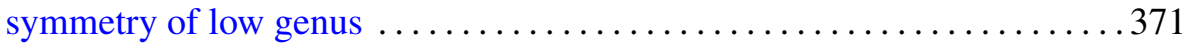

Louis Jackson Ratliff, Jr., On asymptotic prime divisors . . . . . . . . . . . 395

Norbert Riedel, Disintegration of KMS-states and reduction of standard von

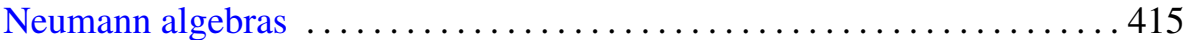

Richard Gordon Swan, $n$-generator ideals in Prüfer domains ...........433

Vilmos Totik, An interpolation theorem and its applications to positive

operators .................................. 447

Richard Vrem, Hypergroup joins and their dual objects 